\title{
The effect of exercise during hemodialysis on serum levels of albumin, calcium, phosphorus and parathyroid hormone: a randomized clinical trial
}

\author{
Alireza Dashtidehkordi ${ }^{\circledR}$, Nahid Shahgholian ${ }^{2 *}$, Jaleh Sadeghian ${ }^{3(}$ \\ ${ }^{1}$ Department of Dialysis, Al-Zahra Hospital, Isfahan University of Medical Sciences, Isfahan, Iran \\ ${ }^{2}$ Kidney Diseases Research Center, Department of Critical Care Nursing, School of Nursing and Midwifery, Isfahan University \\ of Medical Sciences, Isfahan, Iran \\ ${ }^{3}$ Alzahra Research Institute, Isfahan University of Medical Sciences, Isfahan, Iran
}

\section{Correspondence to:}

Nahid Shahgholian E-mail: shahgholian@nm.mui.ac.ir

Received: 30 July 2021 Accepted: 23 Oct. 2021 ePublished: 4 Nov. 2021

Keywords: Exercise, Albumin, Calcium, Phosphorus, Parathyroid hormone, Hemodialysis

\begin{abstract}
Introduction: Disorders in the levels of blood electrolytes are often considered as complications of kidney illnesses which are responsible for various systemic problems experienced by patients. Objectives: The aim of this study was to investigate the efficiency of an exercise program during hemodialysis on serum levels of biochemical factors such as albumin, calcium, phosphorus and intact parathyroid hormone (iPTH).

Patients and Methods: Sixty patients undergoing hemodialysis were evaluated in the clinical trial. The patients in the intervention group pedaled on a stationary bicycle for one hour in every hemodialysis session for eight weeks. At the beginning of the study and at the end of it, the patients' blood samples were sent to hospital's laboratory to measure the levels of biochemical factors in blood. Data were analyzed by SPSS software.

Results: The serum levels of electrolytes showed no significant improvement between the study groups before and after the exercise program $(P>0.05)$. A significant improvement was observed after the program in serum phosphorus and iPTH $(P=0.04)$. In contrast, serum albumin $(P=0.3)$ and calcium $(P=0.5)$ did not alter significantly after the exercise program.

Conclusion: Exercise improved some biochemical factors of blood in the patients undergoing hemodialysis. Exercise is therefore a complementary procedure for these patients.

Trial Registration: The trial protocol was approved in the Iranian Registry of Clinical Trial (\#IRCT20150116020675N4; https://en.irct.ir/trial/50492, ethical code\# IR.MUI.MED.REC.1399.212).
\end{abstract}

\section{Introduction}

End-stage renal disease (ESRD) is a widespread health problem from which about 50 million people are suffering all around the world. A large percentage of these patients require hemodialysis as a complementary method of treatment (1). The United States Renal Data System has announced that the number of patients suffering from ESRD is increasing each year and more than 320000 American patients with ESRD are under treatment with hemodialysis (2). In many other countries, the high incidence and prevalence of the disease has been reported. Hemodialysis as a kind of therapy is essentially required to save the life of about 91.9\% of patients suffering from ESRD (3). Hemodialysis that uses low-flux membranes is not able to remove sufficiently middle molecules. Longer dialysis time and use of high-flux membranes also do not improve clinical outcomes. Albumin loss is another concern in hemodialysis patients.

\section{Key point}

In a randomized clinical trial on sixty patients on hemodialysis, we found exercise improved various biochemical factors, including phosphorous and iPTH in hemodialysis.

Anorexia, insufficient intake of nutrients, nutrient losses during hemodialysis, and elevated protein catabolism contribute to nutritional deficits and hypoalbuminemia in patients undergoing dialysis (4). Hypoalbuminemia is a strong predictor of poor outcome and mortality in these patients. Therefore, interventions are required to improve the levels of albumin (AL) in these patients (5).

One of the most prevalent electrolyte disorders in patients undergoing dialysis is disturbance of calcium and phosphate, which contributes to the development of hyperparathyroidism. Higher amounts of phosphorus, calcium-phosphorus and intact 
parathyroid hormone (iPTH) emerge as important clinical issues in dialysis patients. These electrolyte disorders increase risk of osteoporosis, vascular and cardiovascular diseases in the patients (6). Phosphorus restriction and treatment with calcium $(\mathrm{Ca})$ drugs and other supplements are considered as the most common treatments used to correct the blood levels of calcium and phosphorus. Thus, it is necessary to find more effective treatments for improving these electrolyte disorders $(7,8)$.

One of the effective treatments for the maintenance of electrolyte balance and improvement of mental and physical function and the quality of life of patients undergoing hemodialysis is exercise. Evaluating the effect of exercise on hemodialysis patients, the results of a study suggested that exercise could decrease serum levels of phosphate in the patients, however, it did not have significant effect on serum calcium. Similarly, another study revealed that hyperphosphatemia can be improved in patients using exercise (9). A study by Paluchamy et al also suggested that an exercise program can improve both blood amounts of phosphorous (P) and calcium in patients (10). Kopple et al suggested that exercise during hemodialysis is efficient in improving the amounts of albumin in ESRD patients (11).

\section{Objectives}

This research aimed to investigate the effect of an exercise program on the amounts of calcium, phosphorus and iPTH in the blood of patients with ESRD undergoing hemodialysis.

\section{Patients and Methods}

\section{Study design}

This randomized controlled trial (two-group, two-stage) was conducted in the dialysis wards of two hospitals (Noor and Al-Zahra) located in Isfahan, Iran. Sixty eligible patients undergoing hemodialysis participated in the research. At first, the eligible patients were randomly allocated into the intervention and control groups based on inclusion criteria $(n=30)$, therefore the patients of the two groups could not meet each other.

\section{Inclusion criteria}

Eighteen to sixty-five patients were present in 3-4-hour hemodialysis sessions three times per week, and were being treated for at least three months. None of them had the following problems; a history of parathyroidectomy surgery, ischemic heart disease, myocardial infarction, angina, severe respiratory illnesses that made patients receive oxygen during dialysis, any history of stroke or ischemic attacks during the last three months and also absence of physical disability.

\section{Exclusion criteria}

The presence of diseases such as cardiovascular and pulmonary illnesses as well as musculoskeletal disorders which might prevent the patient from receiving the intervention was one of the inclusion criteria. In addition, the patients who did not do the exercises for three successive sessions or six non-successive sessions and also those who did not have tendency to participate were eliminated from the study.

\section{Data collection}

At the beginning and the end of the intervention, blood samples were taken and delivered to hospital's laboratory to measure serum albumin, calcium, phosphorus and iPTH.

\section{Intervention}

At the beginning of the study, the patients were explained about the exercise program. They started to attend the hemodialysis and began to pedal on stationary bicycle (mini bike). Every session lasted for one hour and with five-minute intervals, and continued for 8 weeks.

The patients themselves chose the rotation the speed of the mini bike. The slowest speed of the mini bike was 15 revolutions per minute, which could be changed by the patients based on their tolerance. Before the intervention and also at the rest intervals, blood pressure of the patients was measured. During the intervention, if the patients faced problems, such as systolic blood pressure of 170 $\mathrm{mm} \mathrm{Hg}$ and higher or lower than $10 \mathrm{~mm} \mathrm{Hg}$, angina and respiratory problems during the exercise, the bicycle was stopped. Moreover, the patients were said to stop the minibike if they felt any exhaustion, headache, palpitations, and other symptoms. The patients of the control group participated in an exercise program, which included 15 stretching movements (any movement lasted for 30 seconds and there was a one-minute interval after each movement). At the end of $8^{\text {th }}$ week, the blood samples were taken and analyzed.

\section{Statistical tests}

The SPSS Statistics software (version 19) was utilized to analyze the obtained data. To compare qualitative variables, including gender, chronic medical conditions and occupational status, the chi-square test was used (Table 1). The independent $t$ test was used to compare quantitative variables, including age, on the duration of dialysis (years), and also the mean amounts of albumin, calcium, phosphorus and iPTH between the two groups. The paired-samples $t$ test was used to compare the mean of serum levels of the variables in each group (Table 2). The minimum significant level was 0.05 .

\section{Results}

Table 1 shows the demographic information of the patients. Out of the 60 patients, 54 patients completed the study period. Of the 30 patients in each group, three patients were excluded (Figure 1). The groups did not show any significant difference in the demographic properties in the pre-test $(P<0.05$; Table 1$)$. In the pre- 
Table 1. Comparison of demographic variables between the two groups

\begin{tabular}{|c|c|c|c|c|c|}
\hline \multirow{2}{*}{ Variables } & & \multicolumn{2}{|c|}{ Control group } & \multirow{2}{*}{$\frac{\text { Intervention group }}{\text { Number }}$} & \multirow{2}{*}{$P$ value } \\
\hline & & Number or Mean & Percent & & \\
\hline \multirow{2}{*}{ Gender } & Female & 24 & & 23 & \multirow{2}{*}{0.61} \\
\hline & Man & 3 & & 4 & \\
\hline \multirow{2}{*}{ Employment status } & Employed & 9 & & 12 & \multirow{2}{*}{0.1} \\
\hline & Unemployed \& retired & 18 & & 15 & \\
\hline \multirow{2}{*}{ Other diseases } & To have & 23 & 22 & & \multirow{2}{*}{0.15} \\
\hline & Not to have & 4 & 5 & & \\
\hline Age & Average age (y) & 55.3 & & 49.9 & 0.1 \\
\hline $\begin{array}{l}\text { The number of months of } \\
\text { treatment with dialysis }\end{array}$ & $\begin{array}{l}\text { Average years of treatment with } \\
\text { dialysis }\end{array}$ & 4.5 & & 5.6 & 0.34 \\
\hline
\end{tabular}

Table 2. Comparison of serum levels of electrolytes before and after the intervention in the intervention and control groups

\begin{tabular}{|c|c|c|c|c|c|c|c|}
\hline \multirow{2}{*}{ Variables } & \multirow{2}{*}{ Times } & \multicolumn{2}{|c|}{ Intervention group } & \multicolumn{2}{|c|}{ Control group } & \multirow{2}{*}{$t$ value } & \multirow{2}{*}{$P$ value } \\
\hline & & Mean & SD & Mean & SD & & \\
\hline \multirow{4}{*}{ Albumin (g/dL) } & Before intervention & 3.87 & 0.4 & 3.98 & 0.4 & 1.04 & 0.3 \\
\hline & After intervention & 4.03 & 0.33 & 3.8 & 0.54 & -1.6 & 0.1 \\
\hline & $P$ value & \multicolumn{2}{|c|}{0.1} & \multicolumn{4}{|c|}{0.17} \\
\hline & $t$ & \multicolumn{2}{|c|}{-1.72} & \multicolumn{4}{|c|}{1.4} \\
\hline \multirow{4}{*}{ Calcium (mg/dl) } & Before intervention & 8.37 & 0.72 & 8.57 & 0.57 & 1.09 & 0.28 \\
\hline & After intervention & 8.53 & 0.56 & 8.6 & .42 & 0.68 & 0.5 \\
\hline & $P$ value & \multicolumn{2}{|c|}{0.14} & \multicolumn{4}{|c|}{0.37} \\
\hline & $\mathrm{t}$ & \multicolumn{2}{|c|}{-1.52} & \multicolumn{4}{|c|}{-0.9} \\
\hline \multirow{4}{*}{$\begin{array}{l}\text { Phosphorus (mg/ } \\
\mathrm{dL} \text { ) }\end{array}$} & Before intervention & 5.08 & 1.01 & 4.91 & 0.1 & -0.6 & 0.55 \\
\hline & After intervention & 4.6 & 1.2 & 5.2 & 0.78 & 2.1 & 0.044 \\
\hline & $P$ value & \multicolumn{2}{|c|}{0.01} & \multicolumn{4}{|c|}{0.22} \\
\hline & $\mathrm{t}$ & \multicolumn{2}{|c|}{2.6} & \multicolumn{4}{|c|}{-1.3} \\
\hline \multirow{4}{*}{$\begin{array}{l}\text { Intact parathyroid } \\
\text { hormone (pg/mL) }\end{array}$} & Before intervention & 470.33 & 315.4 & 456.5 & 334.3 & -0.16 & 0.88 \\
\hline & After intervention & 342.2 & 242.8 & 486.2 & 261.5 & 2.1 & 0.041 \\
\hline & $P$ value & \multicolumn{2}{|c|}{0.003} & \multicolumn{4}{|c|}{0.47} \\
\hline & $\mathrm{t}$ & \multicolumn{2}{|c|}{3.33} & \multicolumn{4}{|c|}{-0.73} \\
\hline
\end{tabular}

test, no significant difference was observed between the two groups in terms of biochemical factors, including albumin, calcium, phosphorus and iPTH. However, after the exercise program, a significant difference was found between the two groups regarding serum phosphorus and iPTH $(P<0.05)$. In this study, no significant difference was observed in the levels of calcium and albumin after the study. In the control group, no statistically significant difference at the end of the study was detected $(P>0.05$; Table 2). Table 3 compares the serum levels of the variables at the beginning and the end of the exercise program in both groups.

\section{Discussion}

Initially, no significant difference was observed in serum levels of biochemical factors of blood between the two groups. However, after the exercise program, significant improvements were found in the levels of some serum electrolytes, including serum phosphate and iPTH. However, significant improvements were not seen on the serum levels of other parameters such as albumin and calcium. In the control group, no significant improvement was observed.

The results of the present study were consistent with the results of the study by Elshinnawy et al, where no significant effect on improving the serum levels of calcium was seen after exercise (12). The results are also in line with the findings of the research conducted by Salhab et al, where they found that an exercise program could be beneficial for hyperphosphatemia patients under hemodialysis (9). Vaithilingam et al, however, showed that exercises during hemodialysis could decrease the serum levels of phosphate, but the decline was not significant. These authors, however, mentioned that exercise reduced the amounts of phosphorus, however significant decrease can be found in long-term use while more intense exercise is needed for a number of participants (13). Our findings are also consistent with the findings of the research conducted by Nesreen et al, since they suggested that changes in calcium metabolism during exercise are dependent on the intensity of exercise. Moderate endurance exercise (for 40 minutes) increases serum calcium level but decreases serum iPTH 


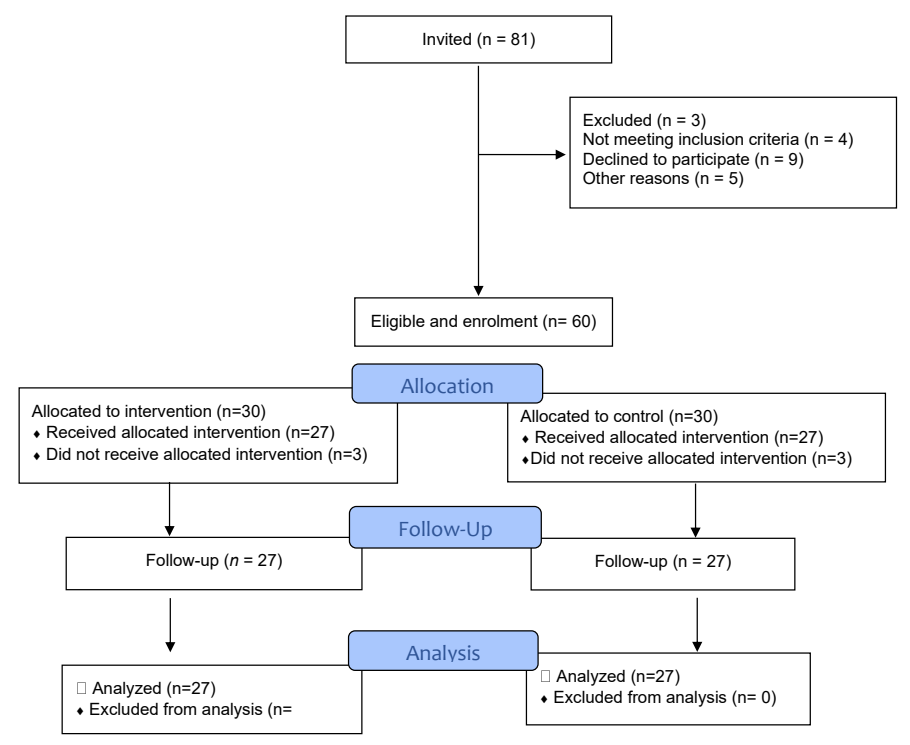

Figure 1. The flowchart of the study.

(14). However, the findings of the present study are not in agreement with the findings of the study of Paluchamy et al, in which they showed an intradialytic exercise program can improve serum levels of both phosphorous and calcium (10). The findings of the present research also are not consistent with the findings of Liao et al, who investigated the impact of aerobic cycling exercise on improving bone density in patients undergoing dialysis. They showed that exercise had no significant effect on improving blood levels of calcium and iPTH in the patients, but it could increase serum levels of albumin (15). Nevertheless, in the present research, no evidence was achieved showing the efficiency of exercise on the patients' levels of albumin in the intervention group compared to the control group. Such inconsistencies might be attributed to the difference in the duration of the intervention (a three-month program) and different exercises (20 minutes riding a bike at the optimal workload). Furthermore, the results of our study are in line with the findings obtained by Kopple et al, who observed that exercise during hemodialysis had no significant effect on serum levels of albumin in the patients of the intervention group (11). The findings of the present research are not in line with the findings of Watanabe et al, where home-based exercise could not significantly affect serum levels of albumin, calcium and phosphate (16). This difference may be related to the use of different exercise programs and the difference in the duration of the exercise (six months compared to eight weeks). The use of minimum sample size and relatively short time of follow-up might be considered as limitations of the study to generalize its findings. In future researches, the use of larger sample sizes and longer periods of followup are suggested.

\section{Conclusion}

According to the results of this research, it can be concluded that an exercise program during hemodialysis can improve blood levels of electrolytes, such as phosphorous and iPTH in these patients. Thus, nurses can use it to decrease electrolyte imbalances that occur in chronic renal failure and to improve general health of the patients.

Limitations of the study

The research findings were limited to the patients with ESRD who were treated in the hemodialysis units of the relevant hospitals.

\section{Authors' contribution}

AD carried out data collection, measurement, statistical analysis, and the interpretation of the results and also wrote the manuscript. He participated in data collection and reviewed medical records. NS participated in reviewing medical records, the study design and coordination as well as managing the research project. All authors

Table 3. Comparison of average difference of serum levels of electrolytes before and after the intervention in both groups

\begin{tabular}{|c|c|c|c|c|c|c|}
\hline \multirow{2}{*}{ Variables } & \multicolumn{2}{|c|}{ Intervention group } & \multicolumn{2}{|c|}{ Control group } & \multirow{2}{*}{$t$} & \multirow{2}{*}{$\boldsymbol{P}$} \\
\hline & Mean & SD & Mean & SD & & \\
\hline Albumin (g/ dL) & 0.03 & 0.52 & 0.18 & 0.56 & 1.5 & 0.15 \\
\hline Calcium (mg/ dL) & 0.14 & 0.57 & 0.18 & 0.87 & 0.24 & 0.8 \\
\hline Phosphorus (mg/dL) & 0.48 & 0.95 & 0.28 & 1.0 & 2.9 & 0.006 \\
\hline Intact parathyroid hormone $(\mathrm{pg} / \mathrm{mL})$ & 128.1 & 200 & 30 & 210 & 2.8 & 0.007 \\
\hline
\end{tabular}


read and approved the final version prior to submission. JS took part in data collection.

Conflicts of interest

The authors declare that they have no competing interests.

Ethics issues

The research was conducted in accordance with the tenets of the Declaration of Helsinki. The Ethics Committee of Isfahan University of Medical Sciences approved this study. The institutional ethical committee at Isfahan University of Medical Sciences accepted all study protocols (ethical code\# IR.MUI. MED.REC.1399.212). Accordingly, written informed consent was taken from all participants before any intervention. The trial protocol was approved by the Iranian Registry of Clinical Trials (identifier: IRCT20150116020675N4; https://en.irct.ir/trial/50492). Additionally, ethical issues (including plagiarism, data fabrication, double publication) have been completely observed by the authors.

\section{Funding/Support}

This study was financially supported by Isfahan University of Medical Sciences (Grant\# 298114).

\section{References}

1. Pretto CR, Winkelmann ER, Hildebrandt LM, Barbosa DA, Colet CF, Stumm EMF. Quality of life of chronic kidney patients on hemodialysis and related factors. Rev Lat Am Enfermagem. 2020;28:e3327. doi: 10.1590/1518-8345.3641.3327.

2. Garg AX, Suri RS, Eggers P, Finkelstein FO, Greene T, Kimmel $\mathrm{PL}$, et al. Patients receiving frequent hemodialysis have better health-related quality of life compared to patients receiving conventional hemodialysis. Kidney Int. 2017:91:746-54. doi: 10.1016/j.kint.2016.10.033

3. McCullough KP, Morgenstern H, Saran R, Herman WH, Robinson BM. Projecting ESRD Incidence and Prevalence in the United States through 2030. J Am Soc Nephrol. 2019;30:127-35. doi: 10.1681/ASN.2018050531

4. Chung S, Koh ES, Shin SJ, Park CW. Malnutrition in patients with chronic kidney disease. Open J Intern Med. 2012;2:89 99; doi: 10.4236/ojim.2012.22018.

5. van Gelder MK, Abrahams AC, Joles JA, Kaysen GA, Gerritsen KGF. Albumin handling in different hemodialysis modalities. Nephrol Dial Transplant. 2018;33:906-913. doi: 10.1093/ndt/ gfx191.

6. Locatelli F, Cannata-Andía JB, Drüeke TB, Hörl WH, Fouque D, Heimburger $\mathrm{O}$, et al. Management of disturbances of calcium and phosphate metabolism in chronic renal insufficiency, with emphasis on the control of hyperphosphataemia. Nephrol Dial Transplant. 2002;5:723-31. doi: 10.1093/ndt/17.5.723.

7. Lombardi G, Ziemann E, Banfi G, Corbetta S. Physical activitydependent regulation of parathyroid hormone and calciumphosphorous metabolism. Int J Mol Sci. 2020;21:53-88. doi: 10.3390/ijms21155388.

8. Moe SM, Drueke TB. Management of secondary hyperparathyroidism: the importance and the challenge of controlling parathyroid hormone levels without elevating calcium،phosphorus،and calcium-phosphorus product. Am J Nephrol 2003;23:369-79. doi: 10.1159/000073945.

9. Salhab N, Alrukhaimi M, Kooman J, Fiaccadori E, Aljubori H, Rizk R, et al. Effect of Intradialytic Exercise on Hyperphosphatemia and Malnutrition. Nutrients. 2019;11:2464. doi: 10.3390/nu11102464.

10. Paluchamy T, Vaidyanathan R. Effectiveness of intradialytic exercise on dialysis adequacy, physiological parameters, biochemical markers and quality of life -a pilot study. Saudi J Kidney Dis Transpl 2018;29:902-10. doi: 10.4103/13192442.239661

11. Kopple JD, Wang H, Casaburi R, Fournier M, Lewis MI, Taylor $\mathrm{W}$, et al. Exercise in maintenance hemodialysis patients induces transcriptional changes in genes favoring anabolic muscle. J Am Soc Nephrol. 2007;18:2975-86. doi: 10.1681/ ASN.2006070794.

12. Elshinnawy, HA, Mohamed AMBB, Farrag DAB, AbdElgawad MAE. Effect of intradialytic exercise on bone profile in hemodialysis patients. Rheumatol Rehabil. 2021;24:48. doi: 10.1186/s43166-021-00071-4.

13. Vaithilingam I, Polkinghorne KR, Atkins RC, Kerr PG. Time and exercise improve phosphate removal in hemodialysis patients. Am J Kidney Dis. 2004;43:85-9. doi: 10.1053/j. ajkd.2003.09.016.

14. El-Nahas NG, Bahey HA, Aboelazm SN. Moderate versus low intensity aerobic exercise on bone mineral density in patients on hemodialysis. Global Journals Inc. 2013;5:11-17.

15. Liao MT, Liu WC, Lin FH, Huang CF, Chen SY, Liu CC, et al. Intradialytic aerobic cycling exercise alleviates inflammation and improves endothelial progenitor cell count and bone density in hemodialysis patients. Medicine (Baltimore). 2016;95:e4134. doi: 10.1097/MD.0000000000004134.

16. Watanabe K, Kamijo $Y$, Yanagi $M$, Ishibashi $Y$, Harada $T$, Kohzuki M. Home-based exercise and bone mineral density in peritoneal dialysis patients: a randomized pilot study. BMC Nephrol. 2021;22:98. doi: 10.1186/s12882-021-02289-y. 